\title{
NOUVELLE
}

\section{Les polykystoses rénales: le gène de la forme récessive enfin identifié}

Simone Gilgenkrantz

famille de protéines très conservée au cours de l'évolution (retrouvée chez l'oursin et chez Coenorhabditis elegans). Puis, 3 ans

9, rue Basse, 54330 Clerey-sur-Brenon, France.
> Le caractère héréditaire des polykystoses rénales (polycystic kidney disease, PKD), maladies génétiques fréquentes et de pronostic grave, fut évoqué dès le XIXe siècle. Vers 1960, la distinction entre PKD autosomiques dominantes $(A D)$ et PKD autosomiques récessives $(A R)$ fut clairement définie. La forme ADPKD se manifeste le plus souvent à l'âge adulte, tandis que la forme ARPKD se manifeste dans l'enfance. Entre 1994 et 1997, médecine/sciences n'a pas manqué de faire part à ses lecteurs des importantes découvertes qui ont permis de comprendre les mécanismes moléculaires des ADPKD. Mais, malgré les efforts déployés et la découverte du locus en 6p21-p12 en 1994, le mystère restait entier pour les formes ARPKD. Le groupe de la Mayo Clinic, avec Peter C. Harris, qui avait déjà participé à l'identification du gène dominant PKDl, vient enfin d'isoler le gène en cause dans les ARPKD.

\section{Formes dominantes et polycystines}

Les polykystoses rénales dominantes sont des maladies extrêmement fréquentes (affectant 1/400 à l/1 000 personnes) dont les premières manifestations cliniques surviennent vers la $\rightarrow)_{m / s} \quad$ troisième ou la quatrième 1994, n6-7, décennie de la vie, mais avec p. $\mathbf{7 6 0}$ une extrême diversité interet même intra-familiale, allant de la découverte de quelques kystes asymptomatiques à une polykystose extensive. Le développement des kystes, la fibrose interstitielle et des phénomènes apoptotiques entraînent une destruction du parenchyme rénal évoluant vers une insuffisance rénale terminale vers l'âge de 50 à 60 ans chez plus de $50 \%$ des sujets atteints [1]. Il existe en outre des manifestations extra-rénales: polykystose hépatique sans gravité (et plus fréquente chez les femmes) et anomalies vasculaires cérébrales à type d'anévrisme.

Deux locus avaient été identifiés : en 16pl3.3p13.12, répondant à $85 \%$ des familles, et en 4 q2123 pour la plupart des autres, permettant une classification en PKDl et PKD2 avec des tableaux cliniques identiques. Quelques rares familles semblent ne correspondre ni à l'un ni à l'autre de ces deux locus et ont été regroupées en PKD3, mais la réalité de ce groupe est incertaine [2].

Il aura fallu 9 ans pour que le gène principal PKDI soit identifié, en grande partie grâce à une translocation $(16 ; 22)$ dans une famille de patients atteints de polykystose rénale $(\rightarrow)$, car sa recherche était rendue difficile par la présence de copies avoisinantes. PKDI comporte 46 exons et code pour une protéine appelée polycystine 1 . Elle appartient à une

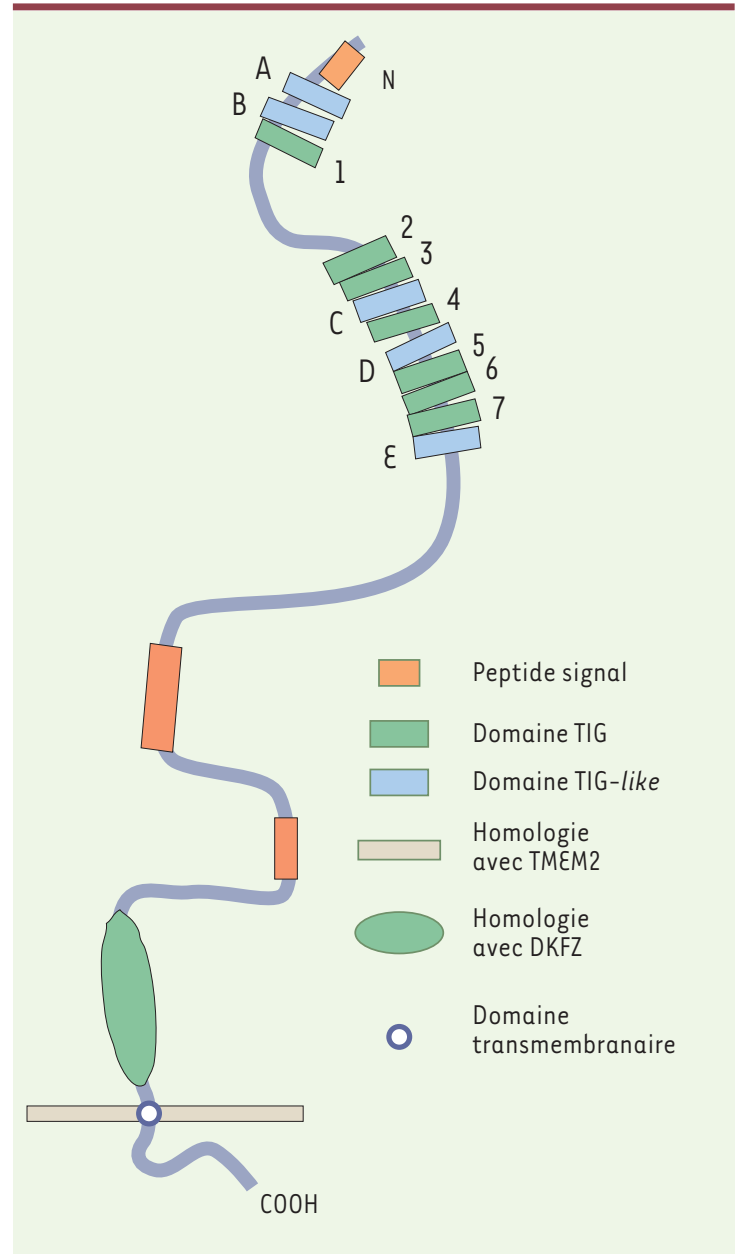

Figure 1. Structure présumée de la fibrocystine. Domaines TIG et TIG-like et régions d'homologie avec d'autres protéines, TMEM2 et DKFZ, de fonction inconnue (d'après [8]). 
règle la morphologie et la fonction tubulaire [3]. Par l'étude des cellules des kystes, qui ont un caractère clonal, il semble que la mutation s'effectue en deux étapes : mutation germinale puis $(\rightarrow) m / s \quad$ mutation somatique $(\rightarrow)$. 1997, n³, Celle-ci peut survenir soit p. 410 dans PKD1, soit dans PKD2, quelle que soit la mutation germinale initiale (état transhétérozygote, connu chez la drosophile, mais qui n'avait pas encore été observé chez l'homme) [4]. Les mutations sont très variées et s'étendent sur toute la longueur des gènes. D'après des études chez le rat, il se pourrait que la tubérine, dont le gène TSC2 est adjacent de PKD1, joue un rôle pour la localisation fonctionnelle de la polycystine 1 et puisse conditionner la gravité de la maladie [5].

\section{Forme récessive et fibrocystine}

Les manifestations cliniques de la polykystose rénale de type récessif se situent à la période périnatale et le diagnostic peut être porté par échographie fœtale avant la naissance, en raison d'un gros rein hyper-échogène, d'un oligoamnios avec hypoplasie pulmonaire consécutive. Les kystes se développent à partir du canal collecteur alors qu'ils se développent à partir de tous les segments tubulaires dans la forme dominante. La forme récessive est constamment associée à une atteinte hépatique, dénommée fibrose hépatique congénitale, fibroadénomatose biliaire ou dysgénésie biliaire. Un diagnostic prénatal peut être réalisé [6]. C'est une des causes majeures de morbidité et de mortalité rénale infantile. Les nouveau-nés peuvent présenter un syndrome de Potter (avec dysmorphie faciale caractéristique). Chez $50 \%$ des enfants qui survivent à la période néonatale, une insuffisance rénale sévère apparaît dans la première décennie de la vie. Toutefois, chez la moitié des enfants qui survivent, l'insuffisance rénale apparaîtra plus tard, après l'âge de 15 ans.

Le locus (qui semble unique) a été identifié il y a plus de sept ans, en 6p21.1- p12, et la recherche du gène fut encore plus difficile que pour les formes autosomiques dominantes.

Pour l'équipe de Peter C. Harris qui vient de l'identifier, l'obtention d'un modèle animal fut déterminante. Plusieurs modèles murins de polykystose rénale avaient été décrits, mais aucun ne correspondait au locus humain. Toutefois, récemment, un rat $p c k$, atteint de polykystose rénale et de fibrose hépatique congénitale a été décrit [7]. La région du locus, sur le chromosome 9 murin, était synténique de la région ARPKD sur le bras court du chromosome 6 humain. II est intéressant de constater que le recours aux programmes de prédiction des gènes a été ici à la fois utile et trompeur : 49 des 67 exons de PKHDI avaient été prédits, mais comme appartenant à 4 gènes, alors qu'en fait, il n'en existe qu'un seul [8]. II manquait 14 exons, et les sites d'épissage sur 4 exons, ainsi que 15 exons, étaient erronés. C'est grâce à la technique RT-PCR, à partir d'ARN de rein humain adulte, qu'un unique transcrit fut obtenu. Le gène PKHDI (pour polycystic kidney and hepatic disease), d'environ $16 \mathrm{~kb}$, est exprimé dans le rein, fœtal et adulte, ainsi que dans le foie et le pancréas adultes. II comporte 67 exons et code pour une protéine appelée fibrocystine. L'implication de PKHDI est confirmée par la mise en évidence, chez 11 malades de familles différentes, de mutations ( 6 tronquantes et 12 fauxsens). Aucun des malades n'était porteur de deux mutations tronquantes, mais 8 d'entre eux étaient des hétérozygotes composites. II est encore trop tôt pour pouvoir établir des corrélations phénotype-génotype. Dans trois familles (le locus étant bien en 6p21), aucune mutation n'a été trouvée. La présence d'un autre gène est peu probable, mais il faut à présent travailler sur de plus grandes cohortes de familles pour exclure une hétérogénéité génétique. II est probable aussi que, dans certains groupes ethniques, en Afrique du Sud par exemple où la maladie est plus fréquente [9], en Espagne [10], ou, à plus forte raison, chez les Druzes (population à forte endogamie) [11], il existe des mutations communes, et des sujets homozygotes pour certaines d'entre elles, ce qui faciliterait le diagnostic prénatal moléculaire.

Quant à la structure et à la fonction de la fibrocystine, il est encore difficile de les définir puisqu'il s'agit d'une nouvelle protéine qui ne correspond à aucune protéine connue (Figure 1). Elle doit posséder un important domaine extracellulaire, avec des domaines TIG et TIG-like, comme les plexines et le HGFR (hepatocyte growth factor receptor). II existe aussi une homologie de séquence avec TMEM2 (transmembrane protein 2), une protéine isolée par hasard, de fonction encore inconnue, qui possède un seul domaine transmembranaire [12]. L'extrémité cytoplasmique de la fibrocystine est courte et possède des sites de phosphorylation. Il est donc possible qu'il s'agisse d'un récepteur intervenant dans la différenciation des tubes collecteurs et des canaux biliaires.

\section{Conclusions}

La découverte du gène PKHDI va faire entrer dans une phase nouvelle les études sur l'ARPKD. La connaissance de la fonction de la fibrocystine sera très précieuse pour comprendre les mécanismes pathogéniques des polykystoses. Le modèle du rat sera aussi très utile pour l'étude des mécanismes pathogéniques au cours du développement et pour tester de nouvelles stratégies thérapeutiques [13]. $\diamond$

Polycystic kidney diseases : a gene identified in autosomal recessive polycystic kidney disease

\section{RÉFÉRENCES}

1. Pirson Y, Chauveau D, Watson ML, Zeier M, Breuning MH. La polykystose autosomique dominante: progrès cliniques et génétiques. Med Sci 1997 ; 13 : 37-44. 
2. Paterson AD, Pei $y$. Is there a third gene for autosomal dominant polycystic kidney disease? Kidney Int 1998 ; 54 : 1759-61.

3. Hanaoka K, Pian F, Boletta $A$, et al. Co-assembly of polycystin-1 and - 2 produces unique cation-permeable currents. Nature $2000 ; 408$ : 990-4.

4. Koptides M, Mean R, Demetriou K, Pierides A, Deltas CC. Genetic evidence of a trans-heterozygous model for cystogenesis in autosomal dominant polycystic kidney disease. Hum Mol Genet 2000 ; 9 : 447-52.

5. Kleymenova $\varepsilon$, IbraghimovBreskrovnaya 0 , Kugoh $\mathrm{H}$, et al. Tuberin-dependent membrane localization of polycystin-1 : a functional link between polycystic kidney disease and the TSC2 tumor suppressor gene. Mol Cell 2001 ; 7 : 823-32.

6. Zerres K, Mucher G, Becker J, et al. Prenatal diagnosis of autosomal recessive polycystic kidney disease (ARPKD) : molecular genetics, clinical experience, and fetal morphology. Am J Med Genet 1998 ; 76: 137-44.

7. Lager DJ, Qian (, Bengal RJ, Ishibashi M, Torres VE. The pck rat: a new model that resembles human autosomal recessive polycystic kidney and liver disease. Kidney Int 2001 ; 59 : 126-36.

8. Ward CJ, Hogan MC, Rossetti $S$, et al. The gene mutated in autosomal recessive polycystic kidney disease encodes a large receptor-like protein. Nat Genet 2002 ; 30 : 259-69.

9. Ramsay M, Reeders ST, Thompson PD, et al. Mutations for the autosomal recessive and autosomal dominant forms of polycystic kidney disease are not allelic. Hum Genet $1988 ; 79: 73-5$.

10. Martinez-Frias ML, Bermejo $\varepsilon$, Cereijo A, Sanchez $M$, Lopez M, Gonzalo C. Epidemiological aspects of mendelian syndromes in a Spanish population sample. II. Autosomal recessive malformation syndromes. Am J Med Genet 1991 ; 38 : 626-9.

11. Naveh Y, Roguin N,

NOUVELLE

\section{Inactivation du chromosome $X$ chez la souris : les tendances cis et trans pour 2002}

Agnès Bourdet, Claire Rougeulle

$>$ Chez les mammifères, l'existence de chromosomes sexuels hétéromorphes implique la mise en place d'un mécanisme compensateur permettant d'aboutir à une équivalence de dose entre le mâle XY et la femelle XX. Touchant aléatoirement l'un des deux chromosomes $X$ chez la femelle, ce processus, connu sous le nom d'inactivation du chromosome $X$, prend place au cours du développement embryonnaire précoce et se caractérise, in fine, par l'extinction transcriptionnelle de la quasi-totalité des quelque deux mille gènes portés par le gonosome touché. Proposé dès 1961, ce mécanisme de régulation génique concertée laisse encore la part belle au mystère. Cependant, les mécanismes moléculaires qui sous-tendent les phases précoces de ce phénomène sont aujourd'hui de plus en plus décortiqués et plusieurs éléments clés ont été caractérisés [1].

Le centre d'inactivation ( $\mathrm{X} i \mathrm{c}$ ), région unique du chromosome $X$, est impliqué dans les étapes précoces de l'inactivation, en particulier le comptage du nombre de chromosomes $X$ présents dans la cellule et le choix du chromosome à inactiver. Le Xic est également le site à partir duquel l'inactivation se propage en cis le long du chromosome X.
Unité de Génétique Moléculaire Murine, Institut Pasteur,

25 , rue du Docteur Roux, 75015 Paris, France.

Identifié au sein du Xic, le gène Xist engendre un transcrit nucléaire de grande taille, issu exclusivement du chromosome $X$ inactif $(X i)$, lequel s'en trouve entièrement recouvert. Des expériences d'invalidation ont démontré le rôle majeur de ce gène dans l'inactivation, mais d'autres acteurs entrent également en jeu [2]. C'est le cas de Tsix, un gène antisens de Xist qui semble impliqué dans la régulation de ce dernier [3] $(\rightarrow)$. L'ARN Xist pourrait ensuite recruter des facteurs de nature encore incon- $(\rightarrow) \mathrm{m} / \mathrm{s}$ 2000, n०6-7, p. 818 